\title{
Advancement in heterogeneous base catalyzed technology: an efficient production of biodiesel fuels
}

\begin{abstract}
Price fluctuation of petroleum-based diesel, climate change, emerging mandate obligations, availability of new feedstock and the upgrading of conversion technologies are expected to drive biodiesel market to grow robustly in the next coming 10 years. However, the current bottleneck in biodiesel production is the lack of economical sustainable conversion technologies. Generally, industrial production of biodiesel is greatly relied on alkaline homogeneous transesterification reaction. Limitation of the technology, such as multistep process which incur extra pre-step for high acid oil treatment and post-step for biodiesel purification and alkali washing as diminished the economic feasibility and low environmental impact of the entire biodiesel process. Heterogeneous catalysis offers immense potential to develop simple transesterification process, including one step reaction, easy separation, reusability of catalyst, and green reaction. Thus, the aim of this paper is to review the biodiesel production technologies such as blending, micro-emulsion, pyrolysis, and transesterification. Furthermore, recent studies on heterogeneous catalyzed transesterification were presented by discussing the issues such as catalytic performance on different types of biodiesel feedstock, transesterification reaction conditions, limitations encountered by heterogeneous catalysts, and reusability of solid catalysts. The heterogeneous catalysts presented in this review is mainly focused on solid base catalysts, which include single metal oxides, supported metal oxide, binary metal oxide, hydrotalcite, and natural waste shell-based catalyst. Furthermore, current perspectives on application of heterogeneous catalyzed technology in biodiesel industry were discussed herein.
\end{abstract}

\section{Keyword:}

